\title{
A siRNA screen reveals the prosurvival effect of protein kinase $A$ activation in conditions of unresolved endoplasmic reticulum stress
}

\author{
MA Aguileta ${ }^{1,2}$, D Rojas-Rivera ${ }^{1,2}$, V Goossens ${ }^{1,2}$, Y Estornes ${ }^{1,2}$, G Van Isterdael $^{1,2}$, P Vandenabeele ${ }^{1,2}$ and MJM Bertrand ${ }^{\star, 1,2}$
}

The endoplasmic reticulum (ER) has a crucial role in the proper folding of proteins that are synthesized in the secretory pathway. Physiological and pathological conditions can induce accumulation of mis- or unfolded proteins in the ER lumen and thereby generate a state of cellular stress known as ER stress. The unfolded protein response aims at restoring protein-folding homeostasis, but turns into a toxic signal when ER stress is too severe or prolonged. ER stress-induced cellular dysfunction and death is associated with several human diseases, but the molecular mechanisms regulating death under unresolved ER stress are still unclear. We performed a siRNA-based screen to identify new regulators of ER stress-induced death and found that repression of the Carney complex-associated protein PRKAR1A specifically protected the cells from ER stress-induced apoptosis, and not from apoptosis induced by etoposide or TNF. We demonstrate that the protection results from PKA activation and associate it, at least in part, with the phosphorylation-mediated inhibition of the PKA substrate Drp1 (dynamin-related protein 1). Our results therefore provide new information on the complex regulation of cellular death under ER stress conditions and bring new insights on the conditions that regulate the pro- versus anti-death functions of PKA.

Cell Death and Differentiation (2016) 23, 1670-1680; doi:10.1038/cdd.2016.59; published online 24 June 2016

The endoplasmic reticulum (ER) is a cellular organelle in which membrane and secreted proteins are synthesized and matured. Many cellular conditions can alter proper ER functions, resulting in the accumulation of un- or misfolded proteins within the organelle lumen, and thereby creating a state of cellular stress called ER stress. Eukaryotic cells have developed a quality control system, known as the unfolded protein response (UPR), to sense and adapt to ER stress. ${ }^{1}$ In metazoans, the UPR is activated by three ER-anchored receptors (inositol-requiring protein 1 (IRE1), protein kinase RNA-like ER-kinase (PERK) and activating transcription factor 6 (ATF6)) that sense an increase of misfolded proteins in the ER lumen and activate cytosolic signaling pathways aimed at restoring protein homeostasis by reducing the protein load. These pathways are activated in a dynamic manner. The UPR promotes the transcriptional upregulation of genes involved in protein folding and later blocks cap-dependent mRNA protein synthesis and promotes protein degradation by ERassociated degradation and autophagy. ${ }^{1}$ Although crucial for restoring homeostasis to protein folding in the ER, the UPR is probably not the only cellular response to ER stress. In budding yeast, ER stress was recently reported to induce protein kinase $A(P K A)$ deactivation, which results in the transcriptional upregulation of stress-related genes and repression of ribosome biogenesis genes. ${ }^{2}$ PKA deactivation would therefore, at least in yeast, also reduce the protein load by affecting the translational capacity of the cell.

When ER stress is too severe or prolonged, the UPR is unable to restore protein-folding homeostasis and consequently turns into a toxic signal that commits the cell to die. ${ }^{1,3,4}$ Cell death caused by unresolved ER stress is a complex process that remains poorly understood. Results from previous studies indicate that the activated cell death pathway and the resulting cell death modality depend on the cell type, on the cellular context and possibly also on the source of ER stress inducer. Indeed, although widely reported to induce caspase-dependent apoptosis by activating the mitochondrial intrinsic pathway, unresolved ER stress is also reported to activate the death receptor extrinsic apoptotic pathway or even to kill cells by necrosis or necroptosis, a regulated form of necrosis. ${ }^{5-7}$ In the case of mitochondrial apoptosis induction, it is reported that signals emerging from the three branches of the UPR (IRE1, PERK and ATF6) converge, or contribute separately, to the dismantlement of the cell. ${ }^{6}$ Activation of the intrinsic apoptotic pathway relies on the BAX/BAK (B-cell lymphoma 2 (BCL-2)-Associated X protein/BCL-2 Antagonist/ Killer)-dependent induction of mitochondrial outer membrane permeabilization (MOMP), which causes cytochrome $c$ release and assembly of the apoptosome, the procaspase 9-activating platform. The three UPR sensors are reported

${ }^{1}$ Inflammation Research Center, VIB, Ghent University, Technologiepark 927, Zwijnaarde-Ghent 9052, Belgium and ${ }^{2}$ Department of Biomedical Molecular Biology, VIB, Ghent University, Technologiepark 927, Zwijnaarde-Ghent 9052, Belgium

*Corresponding author: MJM Bertrand, Department of Biomedical Molecular Biology, VIB, Ghent University, Technologiepark 927, Zwijnaarde-Ghent 9052, Belgium. Tel: +3293313720; Fax: +3293313511; E-mail: mathieu.bertrand@irc.vib-ugent.be

Abbreviations: ER, endoplasmic reticulum; UPR, unfolded protein response; MEF, mouse embryonic fibroblast; IRE1, inositol-requiring protein 1; PERK, protein kinase RNA-like ER kinase; ATF6, activating transcription factor 6; CHOP, C/EBP-homologous protein; TNF, tumor necrosis factor; PKA, protein kinase A; BAX, B-cell lymphoma 2 (BCL-2)-associated X protein; BAK, BCL-2 Antagonist/Killer; MOMP, mitochondrial outer membrane permeabilisation; XBP-1, X-box-binding protein 1; Drp1, dynaminrelated protein 1; CHX, cycloheximide; Frsk, forskolin; AMPK, AMP-activated protein kinase- $\alpha$; Mdivi-1, mitochondrial division inhibitor-1

Received 14.12.15; revised 10.5.16; accepted 30.5.16; Edited by SH Kaufmann; published online 24.6.2016 
capable of inducing MOMP by modulating expression and/or activation of various BCL-2 family members via distinct mechanisms. ${ }^{8}$ Concomitantly with MOMP and cytochrome $c$ release, mitochondria have been reported to undergo dynamin-related protein 1 (Drp1)-dependent fission during apoptosis, but whether mitochondrial fragmentation is required for apoptosis induction has long been a subject of controversy. $^{9}$

Cell death is an important biological outcome that protects the organism from accumulating damaged or unnecessary cells. Nevertheless, cell death needs tight regulation because inappropriate cell death induction drives various human pathologies, such as cancers or neurodegenerative disorders. ${ }^{10,11}$ In this study, we performed a small-scale siRNA-based screen to identify new regulators of ER stressinduced death. Among the 'hits' obtained, we further characterized PRKAR1A, a regulatory subunit of PKA. PRKAR1A was identified as a tumor suppressor, due to the fact that mutations in PRKAR1A, which lead to PKA activation, cause an autosomal dominant disease called Carney complex, a disorder characterized by hyperpigmentation of the skin, cardiac and other myxomas, endocrine tumors and schwannomas. ${ }^{12,13}$ Paradoxically, Prkar1a ${ }^{-/}$mice die during embryogenesis, and the lethality has been associated with an increase in apoptosis. ${ }^{12,14-17}$ Interestingly, we found (I) that PRKAR1A repression specifically protected cells from apoptosis induced by ER stress and not by other apoptotic triggers, (II) that this protection is due to PKA activation and (III) that it is partially attributed to Drp1 phosphorylation and consequent inactivation. Taken together, our results provide new information on the complex regulation of ER stress-induced apoptosis and, by linking a prosurvival function of PKA to ER stress conditions, bring a cellular context for the paradoxical prosurvival versus prodeath role of PKA, which can be of relevance in the case of the Carney complex disorder.

\section{Results}

A siRNA screen identifies new modulators of ER stressinduced cell death. To identify new regulators of cell death induced by ER stress, we carried out a screen on mouse embryonic fibroblasts (MEFs) using an in-house library of SMARTpool siRNAs targeting 275 distinct genes that had previously been associated, closely or from further, with cell survival/death decisions in different signaling pathways (Supplementary Tables 1 and 2). ER stress-induced death in MEFs was obtained by tunicamycin (Tu) treatment and analyzed $18 \mathrm{~h}$ poststimulation, a timing allowing $30-45 \%$ cell death in the nonspecific (NS) siRNA-transfected cells (Figure 1a). The extent of cell death was determined by high content imaging (BDPathway 855 instrument, BD Biosciences, San Jose, CA, USA) of the nuclear stains Hoechst and propidium iodide $(\mathrm{PI})$. Hoechst is cell permeable and used to stain all nuclei, while $\mathrm{PI}$ is cell impermeable and therefore used to stain the cells with ruptured plasma membrane. The percentage of cell death was therefore defined as the percentage of cells positive for both staining (Figure 1a). We previously showed that Tu treatment induces apoptosis in MEFs and therefore used caspase-3-targeting
SiRNAs as a positive control (Figure 1a). ${ }^{18}$ The workflow of the screen consisted in knockdown (KD) of each set of genes in 6-plicate plates, having every gene $\mathrm{KD}$ in six different plates. Three plates were stimulated with $\mathrm{Tu}$, and the others with DMSO. This setup allowed us to discern whether the KD of any given gene had an impact on cell viability on its own. None of the targeted gene had a significant effect on basal cell viability, except for Matk, Rbx1 and Ppp1cb that, respectively, led to $14 \%, 13 \%$ and $7 \%$ cell death upon repression, respectively. Both positive (Casp 3) and negative (NS) controls were included in each plate. Because the Tuinduced cell death varied between screening days, we normalized the percentage of Tu-induced cell death for each gene to the plate-specific NS control siRNA (Figure 1b). To identify genes positively involved in the ER stress-induced cell death pathway, genes providing at least $30 \%$ protection upon repression were selected and defined as 'hits' (Figures $1 \mathrm{~b}$ and $\mathrm{c}$ and Supplementary Tables 1 and 2). This led us to the identification of 22 genes (Figure 1c), out of which 7 were validated by kinetic cell death analysis measuring nuclear SytoxGreen fluorescence in death cells (Fluostar Omega fluorescence plate reader, BMG Labtech, Ortenberg, Germany), as previously described ${ }^{19}$ (Figures 1d-j and Supplementary Figure 1). Of note, two of these seven genes, Pmaip1 (Noxa) and Bad, are reported mediators of ER stress-induced death, ${ }^{20,21}$ confirming the validity of our screen.

Prkar1a depletion protects against ER stress-induced apoptosis. Among the five newly identified positive regulators of ER stress-induced death (Prkar1a, Ube2v1, Rnf11, Zranb1 and Atp5a), we decided to further characterize Prkar1a, a regulatory subunit of PKA linked to the Carney complex disorder in human. We found that the protection to Tu-induced cell death obtained by siRNA-mediated repression of Prkar1 in MEFs was associated with reduced caspase-8, -9 and -3 processing as well as caspase- 3 activity (Figures $2 \mathrm{a}-\mathrm{c}$ ), indicative of reduced apoptosis induction. Caspase inhibition by the pancaspase inhibitor z-VAD-fmk greatly, but not entirely, protects MEFs from Tu-induced death (Supplementary Figures 2A and B). To test whether Prkar1a repression also protects MEFs from caspase-independent death, we stimulated the cells with $\mathrm{Tu}$ in the presence of z-VAD-fmk and observed no additional protection (Supplementary Figure 2C). To exclude any off-target effect of the Prkar1a-targeting siRNA, we confirmed our results in Prkar1a $^{-1-}$ MEFs. To obtain these cells, Prkar1a ${ }^{f l / f l}$ MEFs $^{22}$ were immortalized using SV40 large T antigen and transduced with adenovirus expressing GFP or GFP-Cre recombinase. Following FACS sorting for GFP positivity, we obtained Prkar1a ${ }^{\text {fl/fl }}$ (GFP) and Prkar1a ${ }^{-/}$(GFP-Cre) MEF cultures. As shown in Figure 2, genetic deletion of Prkar1a in MEFs conferred protection to Tu-induced apoptosis similarly as following siRNA-mediated repression of Prkar1a (Figures 2d-f). Importantly, the protection was not limited to Tu treatment but was also observed following thapsigargin (Th) stimulation, another commonly used ER stress inducer (Figures $1 \mathrm{~g}$ and $\mathrm{h}$ ). In line with this, we found that the protection was not cell type or species specific, as PRKAR1A repression also protected human HeLa cells from Tu- and 
a

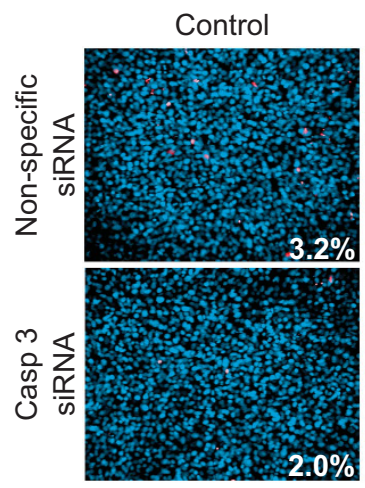

Hoechst/PI
ER stress (Tu)

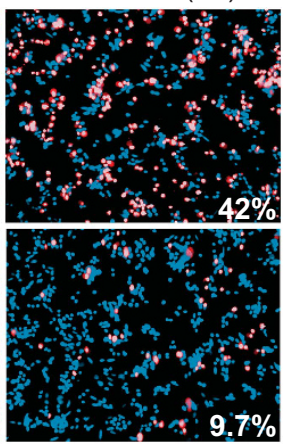

Hoechst/PI

\section{b}

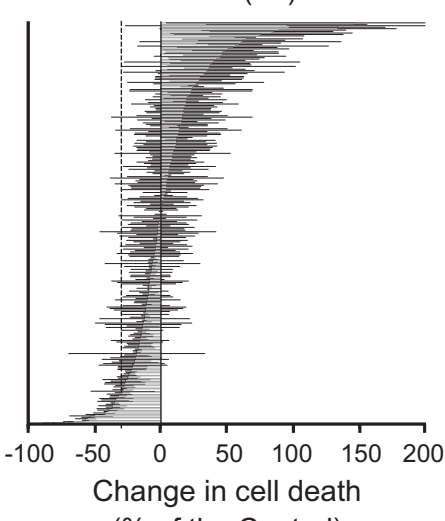

(\% of the Control)

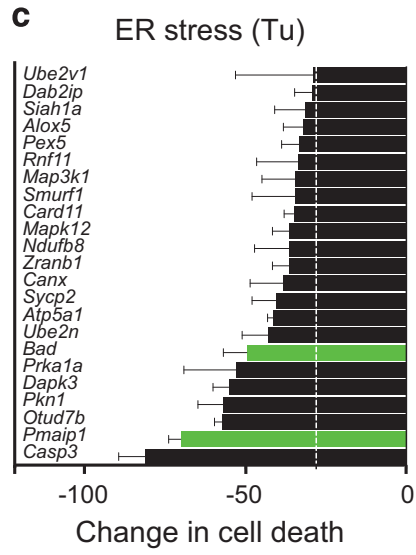

(\% of the Control)

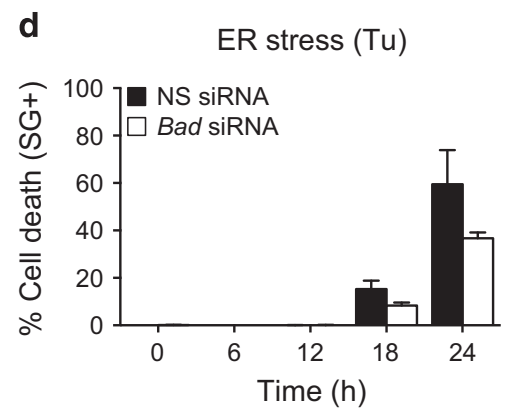

e

ER stress (Tu)

f

g ER stress (Tu)

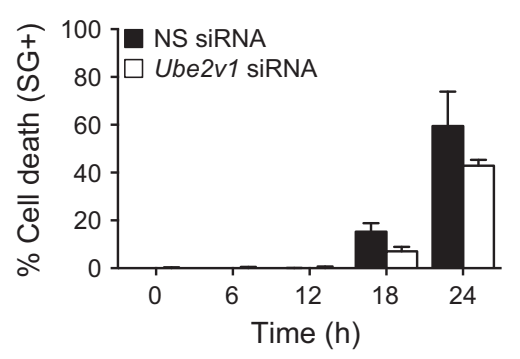

j

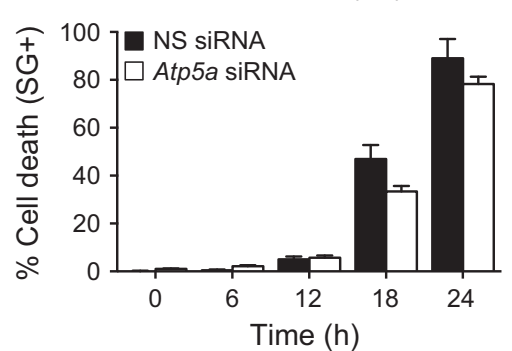

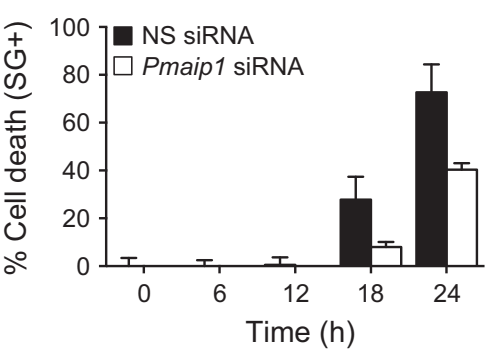

h

ER stress (Tu)

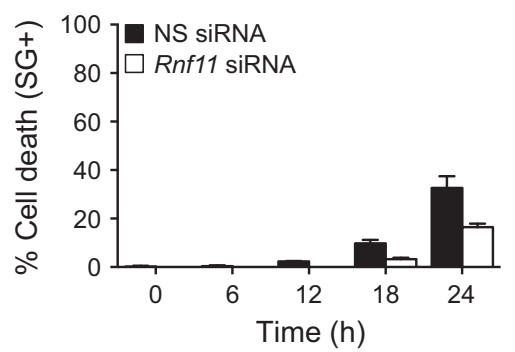

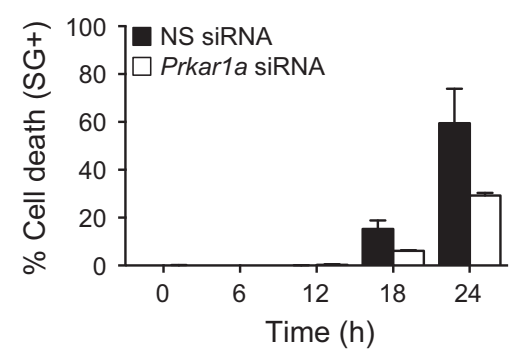

i

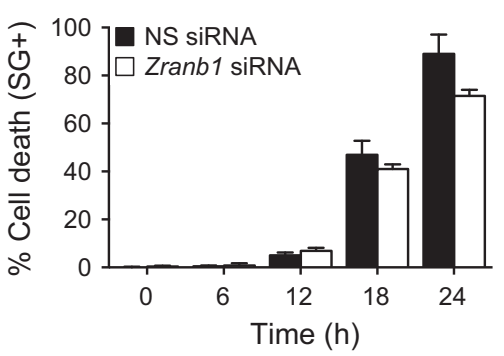

Figure 1 A siRNA screen identifies new modulators of ER stress-induced cell death. (a) Representative images showing Hoescht and PI staining of MEFs transfected with nonspecific (NS) siRNA or with siRNA targeting caspase-3 and stimulated, or not, with Tu for $18 \mathrm{~h}$. The percentage of cell death is calculated as the percentage of cells doubly positive for Hoescht and PI. (b) Schematic representation of the results from the screen. The line shows the arbitrary cutoff in the genes providing at least $30 \%$ protection upon repression. The names of these genes are shown in (c) and the ones highlighted in green are the previously reported ones. The graphs show the mean \pm S.D. of one experiment carried out in triplicate. (d-j) WT MEFs transfected with the mentioned siRNAs were stimulated with Tu, and cell death was monitored in function of time by SytoxGreen positivity using the Fluostar Omega fluorescence plate reader. The graphs show the mean \pm S.D. of one experiment carried out in triplicate, and the results are representative of at least two independent experiments 
a

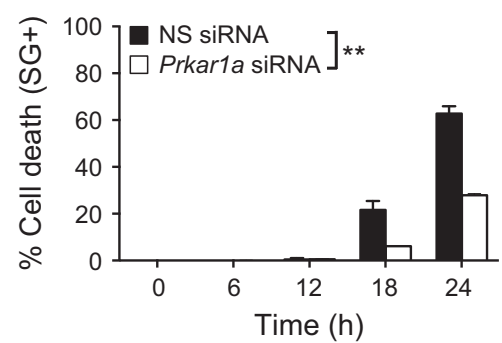

b
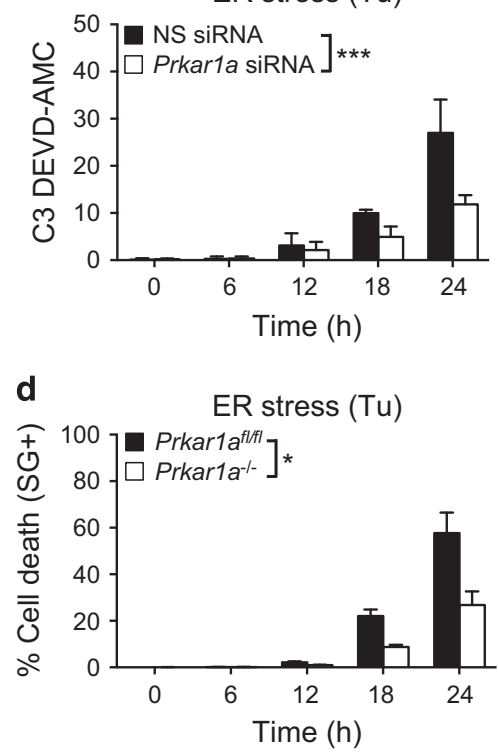

e
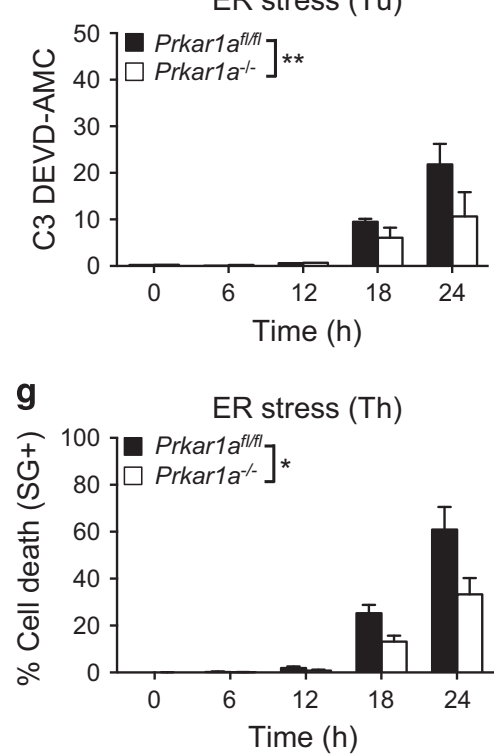

C

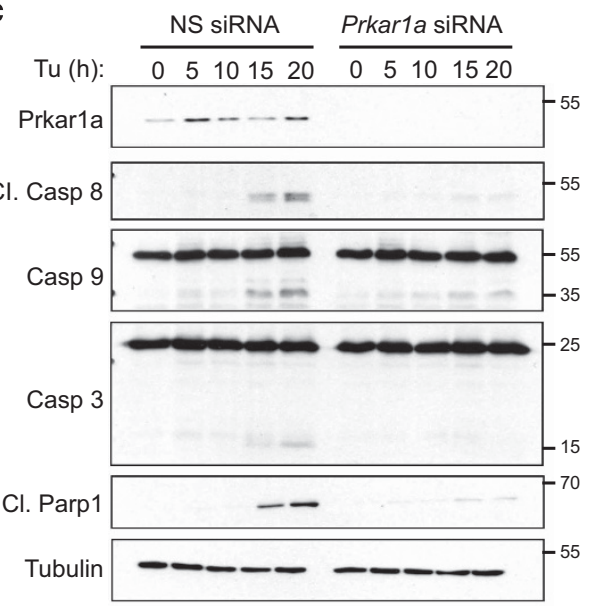

f

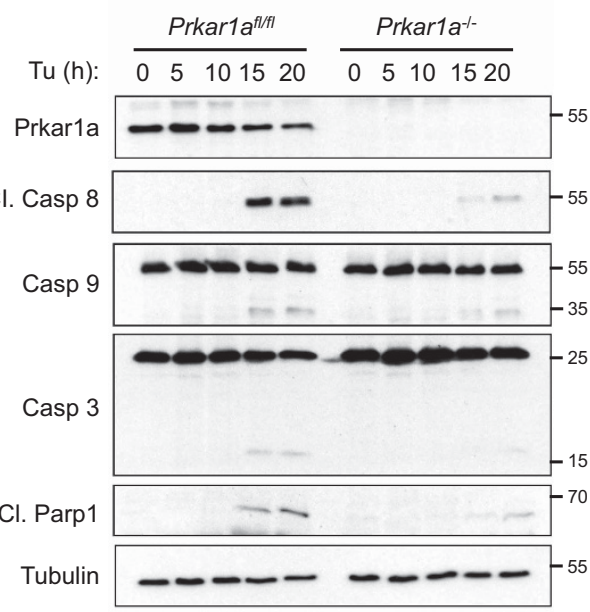

h

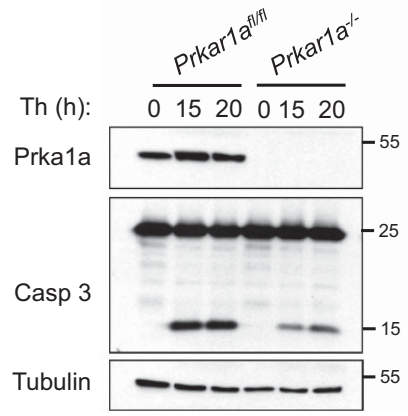

Figure 2 Prkar1a depletion protects against ER stress-induced apoptosis. (a-c) MEFs transfected with control siRNA (NS) or siRNA targeting Prkar1a were stimulated $48 \mathrm{~h}$ later with Tu, and cell death (a) and caspase activity (b) were measured in function of time, respectively, by SytoxGreen positivity and DEVD-AMC fluorescence. (c) Total protein extracts were generated and probed as indicated. (d-f) Prkar1 $a^{f / f t l}$ and Prkar1a ${ }^{-I-}$ MEFs were stimulated with Tu, and cell death (d) and caspase-3 activity (e) were determined as in (a and $\mathbf{b})$. (f) Total protein extracts were generated and probed as indicated. ( $\mathbf{g}$ and $\mathbf{h}$ ) Prkar1a $a^{f / f l}$ and Prkar1a ${ }^{-1-}$ MEFs were stimulated with Th and cell death was determined as in (a). (h) Total protein extracts were generated and probed as indicated. The graphs show the mean \pm S.D. of three independent experiments carried out in triplicate 
Th-induced apoptosis (Supplementary Figures 2D-F). Taken together, these results demonstrate that PRKAR1A depletion protects cells from ER stress-induced apoptosis.

Loss of Prkar1a specifically protects cells from apoptosis under unresolved ER stress conditions. Having established that Prkar1a depletion provides protection to ER stress-induced apoptosis, we next evaluated whether the protection was specific to unresolved ER stress conditions, or also observed when apoptosis is induced by other stimuli. ER stress-induced apoptosis displays characteristics that are found both in the receptor extrinsic apoptotic pathway and in the mitochondrial intrinsic apoptotic pathway, that is, activation of the initiator caspase-8 and -9 , respectively. ${ }^{1,3,4,23}$ Therefore, we decided to engage these two pathways separately and test whether loss of Prkar1a also provides some degree of protection under these specific conditions. Interestingly, we found that Prkar1a $a^{f / f l}$ and Prkar1a ${ }^{-/-}$MEFs responded similarly to apoptosis induced by TNF in combination with cycloheximide $(\mathrm{CHX})$, a classical trigger for activation of the extrinsic apoptotic pathway (Figures $3 a$ and b). To our surprise, the cells also succumbed to a similar extent following activation of the intrinsic pathway by etoposide (Eto) stimulation (Figures $3 c$ and $d$ ). These results therefore indicate that absence of Prkar1a specifically protects cells from ER stress-mediated apoptosis and exclude the possibility that Prkar1a ${ }^{-/}$cells have undergone a physiological change that prevents them from dying under different stimuli.

PKA activation mediates the protection against ER stress-induced apoptosis. PRKAR1A is part of a heterotetramer known as protein kinase $A$ (PKA). This heterotetramer is composed of two heterodimers made of two catalytic subunits (PKAc) and two regulatory subunits (PRKAR). Within the human and mouse genome, there are three variants of $\operatorname{PKAc}(a, \beta$ and $\gamma$ ) and four variants of PRKAR (R1A, R1B, R2A and R2B), with PRKAR1A being the most abundant and ubiquitously expressed. ${ }^{24}$ The function of the regulatory subunits in the PKA complex is to hold the catalytic subunits inactive. Binding of CAMP to PRKAR subunits causes their dissociation from PKAc and results in PKA activation. Physiologically, cAMP is produced when G-protein-coupled receptors are activated upon ligand binding. The $G_{s} a$ subunit of some receptors stimulates adenylyl cyclase to produce cAMP from ATP. We found that PRKAR1A depletion protects cells against ER stress-induced apoptosis, but whether the protection originates from the absence of PRKAR1A or as a consequence of PKA activation is not clear. To answer this question, we took different approaches. First, we tested the effect of PKA depletion, obtained by siRNA, on Tu-induced apoptosis in MEFs. As shown in Figure 4, PKA depletion only slightly sensitized the cells to Tu-induced apoptosis, but completely inhibited the protection
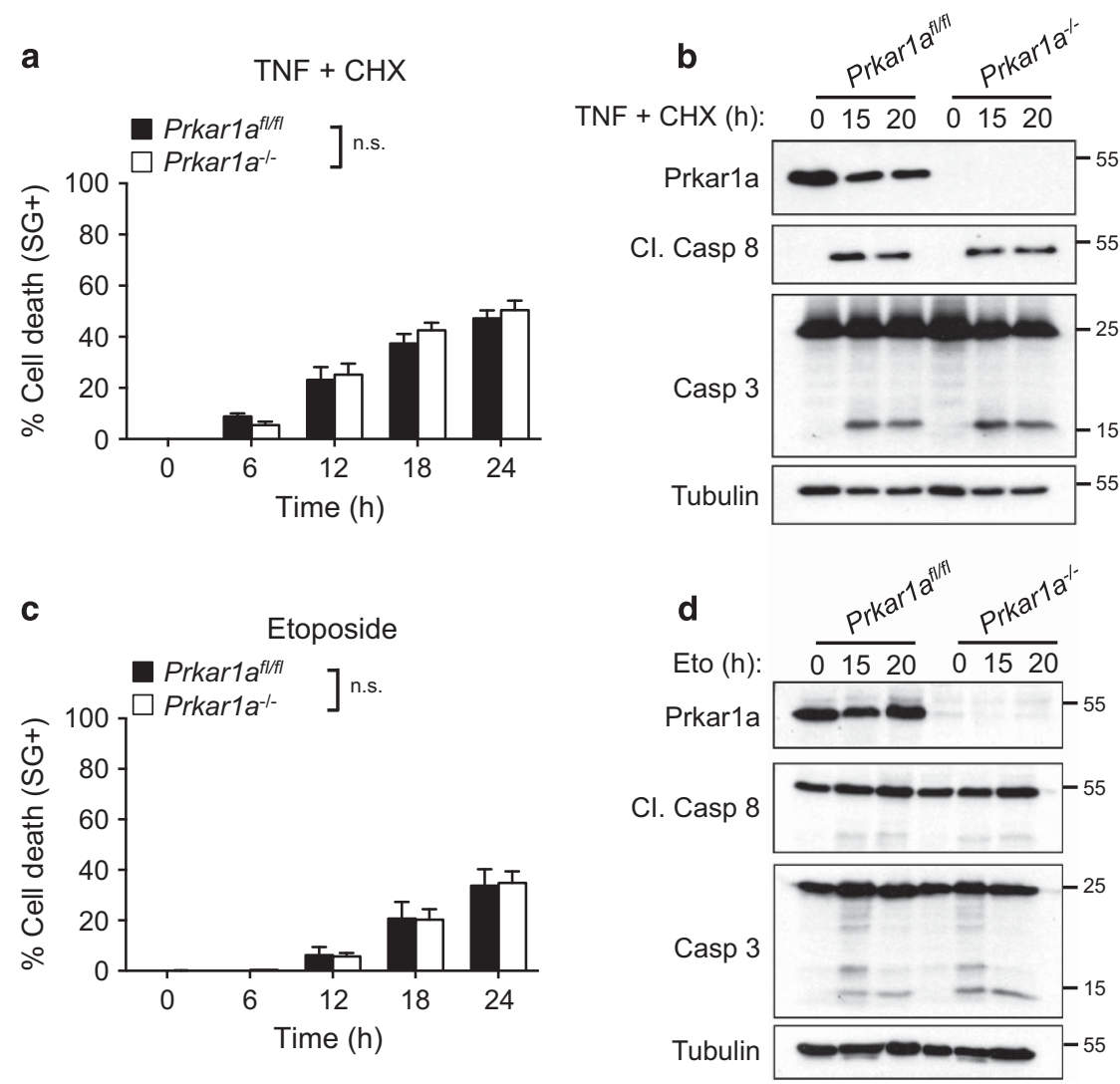

Figure 3 Absence of Prkar1a does not confer resistance to other apoptotic stimuli. (a-d) Prkar1 $a^{f / f t}$ and Prkar1a ${ }^{-l-}$ MEFs were stimulated with TNF in combination with CHX $(\mathbf{a}$ and $\mathbf{b})$ or with Eto ( $\mathbf{c}$ and $\mathbf{d})$. Cell death was monitored in function of time by SytoxGreen positivity using the Fluostar Omega fluorescence plate reader (a and $\mathbf{c})$. Total protein extracts were generated and probed as indicated ( $\mathbf{b}$ and $\mathbf{d}$ ). The graphs show the mean \pm S.D. of three independent experiments carried out in triplicate 
a

ER stress $(\mathrm{Tu})$

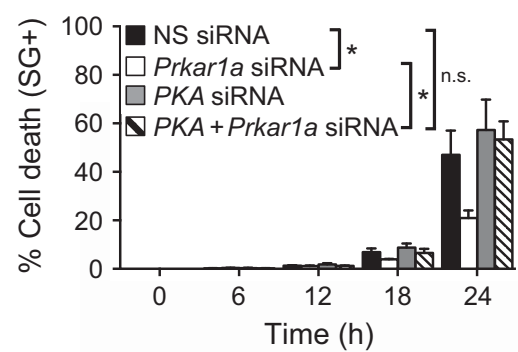

C

ER stress (Tu)

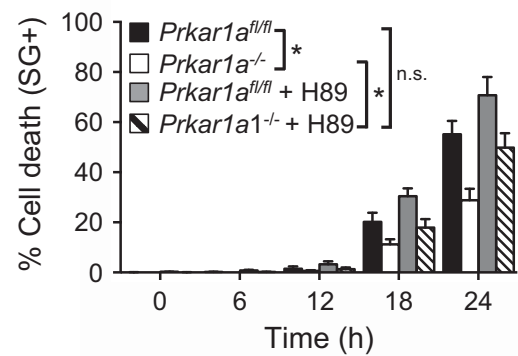

e

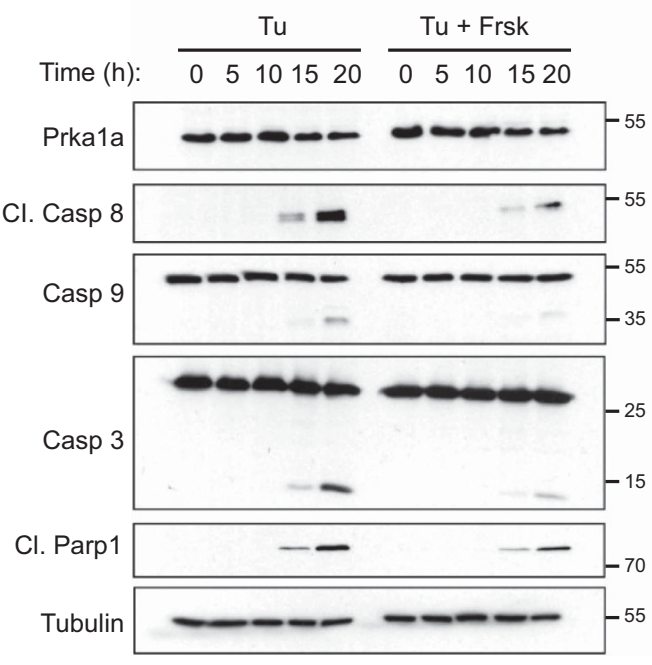

b

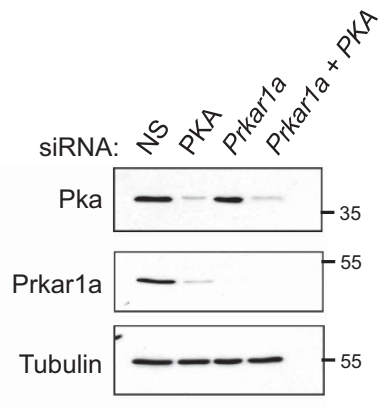

d
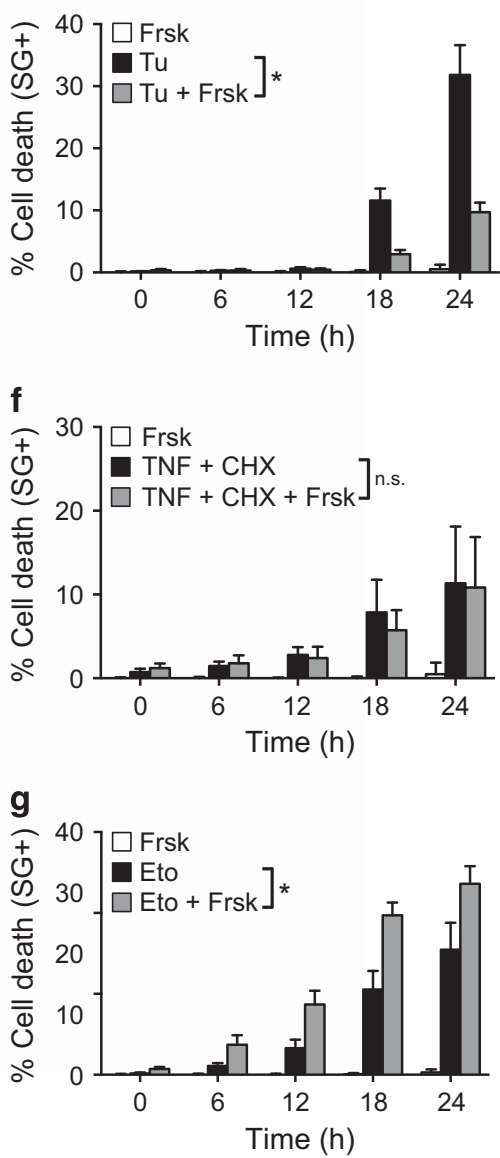

Figure 4 PKA activation protects against ER stress-induced cell death. (a) WT MEFs were transfected with the indicated siRNAs, stimulated with Tu and cell death was monitored in function of time by SytoxGreen positivity using the Fluostar Omega fluorescence plate reader. (b) WT MEFs were transfected as in (a) and cell lysates were generated $48 \mathrm{~h}$ post-transfection and probed as indicated. (c) Prkar1a $\mathrm{f}^{\mathrm{fl} / \mathrm{fl}}$ and Prkar1a ${ }^{-1-}$ MEFs were stimulated with Tu in the absence or presence of the PKA inhibitor H89, and cell death was monitored as in (a). (d-g) WT MEFs were stimulated with $\mathrm{Tu}(\mathbf{d}), \mathrm{TNF}+\mathrm{CHX}(\mathbf{f})$ or Eto (g) in the absence or presence of Frsk and cell death was measured as in (a). (e) Cell lysates were generated at various time points and probed as indicated. The graphs show the mean \pm S.D. of three independent experiments carried out in triplicate

obtained by siRNA-mediated repression of Prkar1a (Figure 4a and Supplementary Figures $3 \mathrm{~A}$ and B). Of note, knocking down PKA caused a reduction in Prkar1a levels, suggesting that Prkar1a is unstable when not in a complex with the catalytic subunits of PKA (Figure 4b). The fact that PKA repression indirectly decreases Prkar1a levels without providing protection against cell death further supports the notion that the protection is mediated by PKA. As a second approach, we treated Prkar1a ${ }^{f / f l}$ and Prkar1a ${ }^{-/-}$MEFs with
Tu alone or in combination with the PKA inhibitor H89. In line with previous results, we observed that chemical inhibition of PKA completely restored the sensitivity of the Prkar1a ${ }^{-1-}$ MEFs to Tu-induced apoptosis (Figure 4c). Intriguingly, we observed that $\mathrm{H} 89$ had some toxicity in Prkar1a ${ }^{f / / f l}$ MEFs but not in Prkar1a ${ }^{-1-}$ MEFs (Supplementary Figure 3C), which explains the higher level of cell death observed in Prkar1a $a^{f / f l}$ MEFs stimulated with Tu and H89 (Figure 4c). Finally, we sought to activate PKA in a more physiological manner and 
took advantage of the well-known adenylyl cyclase agonist Forskolin (Frsk). We challenged WT MEFs with Tu, Frsk or the combination of both, and monitored cell death as well as caspase activation. As shown in Figures 4d-e, PKA activation by Frsk protected the cells from Tu-induced death and was associated with reduced caspase-8, -9 and -3 processing without affecting Prkar1a levels. Importantly, and in line with our previous results, PKA activation by Frsk treatment did not confer protection against cell death induced by TNF in combination with $\mathrm{CHX}$ or by Eto (Figures $4 \mathrm{f}-\mathrm{g}$ ).

PKA activation has no impact on the UPR. Having demonstrated that PKA activation provides protection against ER stress-induced apoptosis, we sought to get further insights on the molecular mechanisms responsible for this protection. Upon release from PRKARs, the free catalytic
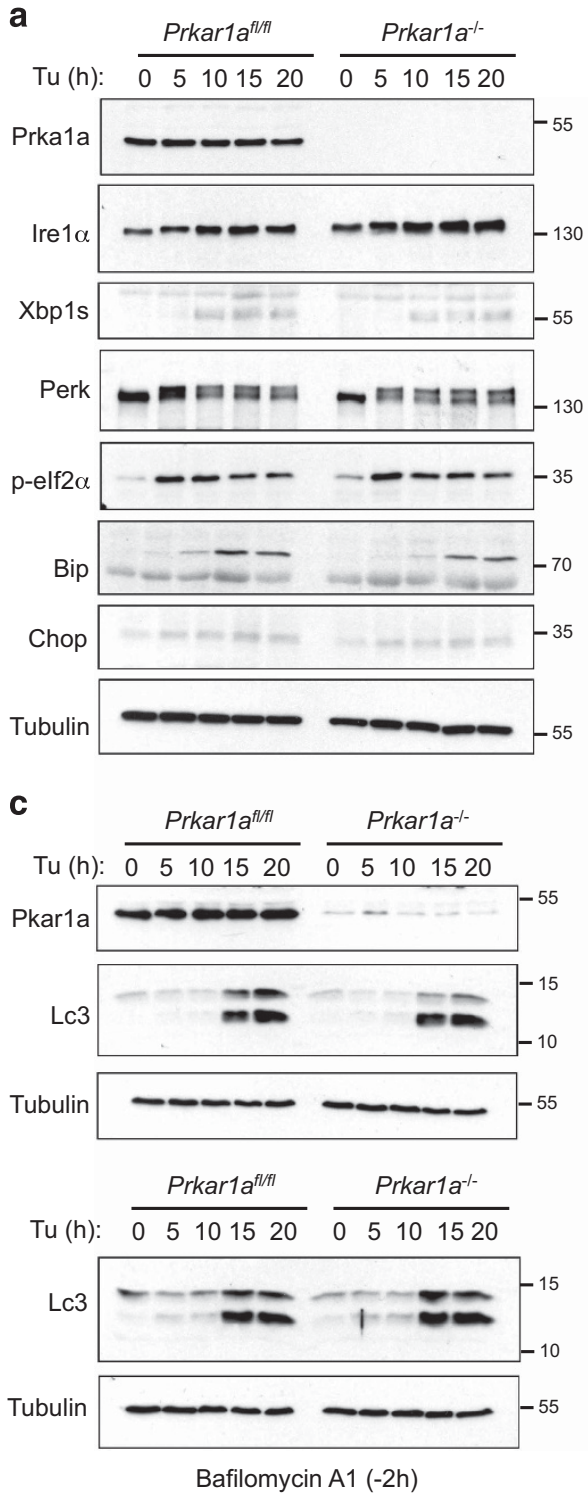

subunits of PKA can affect a wide range of cellular events by phosphorylating a big array of substrates, including transcription factors and enzymes. ${ }^{25,26}$ In addition, PKA activation has been reported to not only prevent but also promote apoptosis, and the molecular explanation for this paradox has not been solved yet. ${ }^{15,27-29}$ Because the UPR has a dual role in the response to ER stress, we decided to first analyze whether PKA activation had any impact on the initiation and/or intensity of the UPR. Using conditions of transient PKA activation (siRNA-mediated repression of Prkar1a) or sustained PKA activation (genetic deletion of Prkar1a), we followed induction of various classical UPR markers over a period of $20 \mathrm{~h}$ of Tu stimulation. We found that PKA had no impact on the activation of the IRE1 or PERK pathway, as monitored by XBP-1 (X-box-binding protein 1) mRNA splicing and phosphorylation of PERK and elf2 $a$, respectively
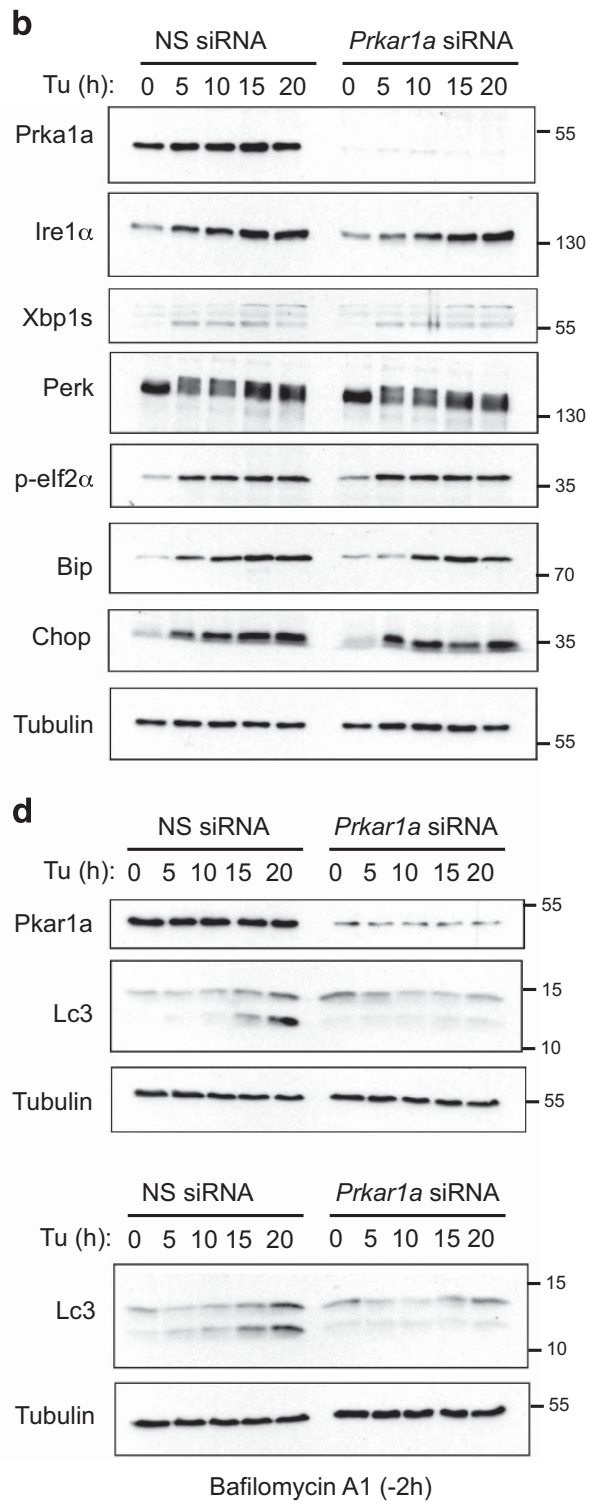

Figure 5 PKA activation has no impact on the UPR. (a-d) Prkar1 $a^{f / f l}$ and Prkar1a ${ }^{-l-}$ MEFs (a and $\left.\mathbf{c}\right)$, or WT MEFs transfected with the indicated siRNAs (b and d), were treated with Tu for various time points and the cell lysates were probed as indicated. (c and $\mathbf{d}$ ) The cells were pre-treated, or not, with bafilomycin $\mathrm{A} 1$ for $2 \mathrm{~h}$ and then stimulated with Tu for the indicated time 
(Figures $5 \mathrm{a}$ and b). In addition, the UPR as a whole did not seem affected as similar induction levels of IRE1, CHOP (C) EBP-homologous protein) and BIP were observed (Figures $5 \mathrm{a}$ and b). Autophagy is a biological consequence of the UPR known to have an important prosurvival role during ER stress. We therefore next tested whether PKA activation could protect cells by boosting autophagy induction in our cellular systems. In contrast, we found that constitutive PKA activation had no impact on autophagy, while transient PKA activation repressed autophagy, as monitored by LC3 lipidation in the absence or presence of bafilomicyn A1 (Figures $5 \mathrm{c}$ and $\mathrm{d}$ ). Taken together, these results indicated that the protective effect of PKA was not resulting from boosted or dampened UPR classic markers.

PKA-mediated protection partially results from Drp1 inhibition. The protective effect seen upon PKA activation can result from phosphorylation of a wide array of substrates, including transcription factors and enzymes. To test whether the protection was the result of activation of a transcription factor, and consequently relying on the transcription and translation of target genes, we evaluated the effect of translation inhibition by $\mathrm{CHX}$ treatment. Interestingly, we found that $\mathrm{CHX}$ treatment protected Prkar1a ${ }^{f / f l}$ MEFs from ER stress-induced death but that PKA activation, obtained by genetic deletion of Prkar1a $\left(\right.$ Prkar1a $\left.^{-/}\right)$, still further protected the cells from death (Figure 6a). These results therefore indicated that PKA protects the cells from ER stress-induced apoptosis independently of translation of survival factors.

Mitochondrial fission, a process regulated by Drp1, is reported to promote apoptosis by contributing to MOMP and cytochrome $c$ release. ${ }^{9,30} \mathrm{PKA}$ and calcineurin were shown to regulate mitochondrial fission and cell death by modulating Drp1 activity through reversible phosphorylation. ${ }^{31}$ PKA inactivates human Drp1 by phosphorylation on Ser637 and abrogates its recruitment to mitochondria. We therefore a

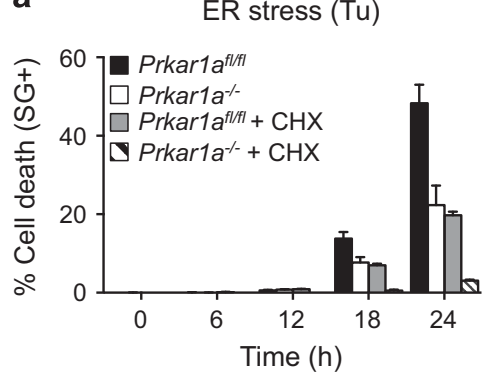

b

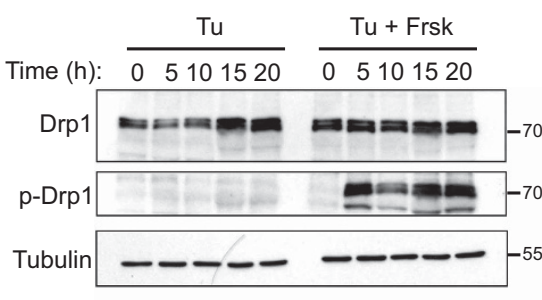

C

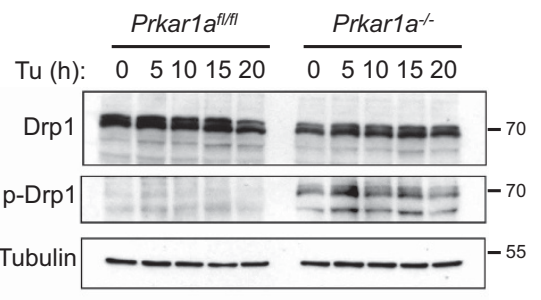

d

ER stress $(\mathrm{Tu})$

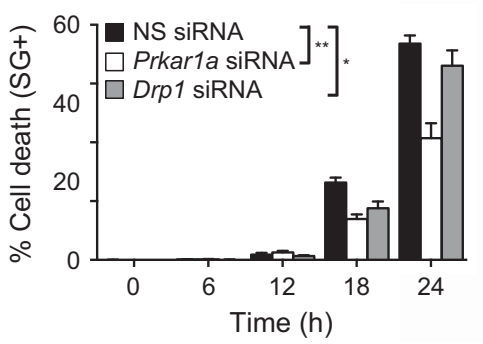

e

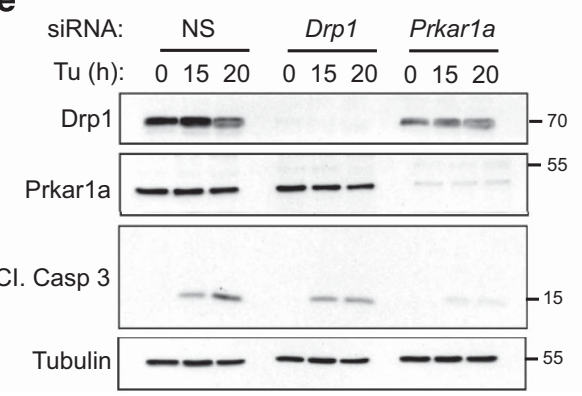

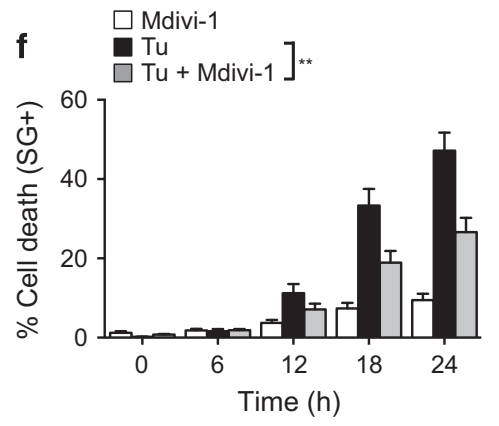
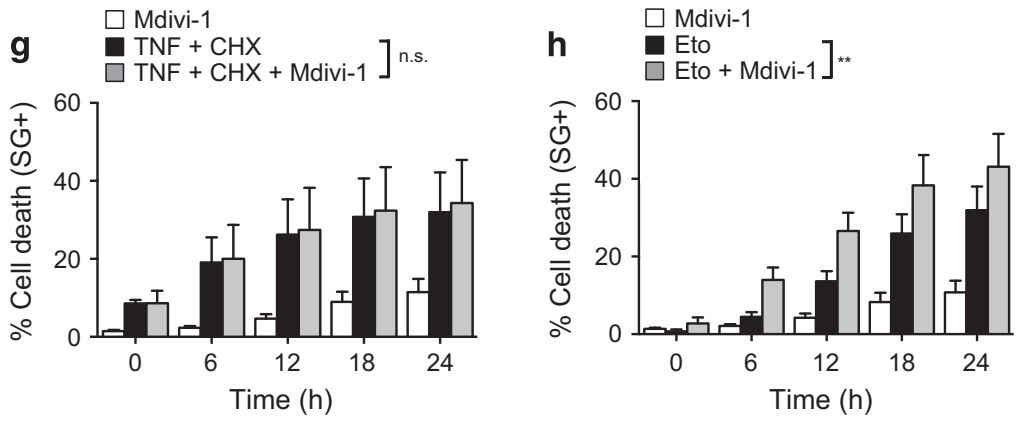

Figure 6 PKA-mediated protection partially results from Drp1 inhibition. (a) Prkar1 $a^{f / f t}$ and Prkar1a ${ }^{-1-}$ MEFs were treated with Tu in the absence or presence of $\mathrm{CHX}$, and cell death was monitored in function of time by SytoxGreen positivity using the Fluostar Omega fluorescence plate reader. (b) WT MEFs stimulated or not with Frsk were treated with Tu for various time points and the cell lysates were probed as indicated. (c) Prkar1at/fll and Prkar1a ${ }^{-l-}$ MEFs were treated with Tu and the cell lysates were probed as indicated. (d) WT MEFs were transfected with the indicated siRNAs, treated with Tu and cell death was measured as in (a). (e) WT MEFs were transfected with the indicated siRNAs, treated with Tu for various time points and the cell lysates were probed as mentioned. $(\mathbf{f}-\mathbf{h})$ WT MEFs cells were stimulated with Tu $(\mathbf{f})$, TNF $+C H X(g)$ or Eto $(\mathbf{g})$ in the presence or absence of Mdivi-1 and cell death was monitored as in (a). The graphs show the mean \pm S.D. of three $(\mathbf{a}, \mathbf{f}-\mathbf{h})$ or five (d) independent experiments carried out in triplicate 
decided to evaluate whether PKA activation in MEFs, obtained via genetic depletion of Prkar1a or following Frsk treatment, leads to Drp1 phosphorylation in our cellular systems. We found that PKA activation was associated with Drp1 phosphorylation in both conditions (Figures $6 \mathrm{~b}$ and $\mathrm{c}$ ). More importantly, we also found that siRNA-mediated repression of Drp1 provided protection, although not to the same extent as Prkar1a, against Tu-induced cell death (Figure 6d). The reduction in cell death was associated with attenuated caspase-3 processing (Figure 6e). The use of mitochondrial division inhibitor-1 (Mdivi-1), a selective inhibitor of Drp1, ${ }^{32}$ further demonstrated the role of Drp1 in ER stress-induced death (Figure 6f). Remarkably, and in line with the absence of protection upon PKA activation, pharmacological inhibition of Drp1 did not provide protection against cell death induced by TNF and $\mathrm{CHX}$ or Eto (Figures $6 \mathrm{~g}$ and $\mathrm{h}$ ).

Altogether, these results indicate that PKA activation selectively protects cells from ER stress-induced apoptosis, at least in part, by phosphorylation-driven inactivation of Drp1, and thereby limiting the activation of the mitochondrial apoptotic pathway.

\section{Discussion}

The ER has a crucial role in the proper folding of proteins that are synthesized in the secretory pathway, and which represent up to $30 \%$ of the whole eukaryotic proteome. Stress in the ER, a possible consequence of a high demand for protein synthesis, is therefore a common feature of specialized secretory cells, and is also associated with the pathogenesis of many human diseases, such as immune disorders, diabetes, cancers and neurodegenerative disorders. ${ }^{10,11,33}$ The UPR, a signaling network initiated by three ER sensors in metazoans, is activated in cells undergoing ER stress. The UPR aims at restoring proper ER function and protein-folding homeostasis. However, when the stress is too severe or prolonged, the UPR initiates signaling cascades resulting in the death of the cell. Because cell death induction may contribute to the pathologies associated with ER stress, we performed a siRNA-based screen to identify new regulators of ER stress-induced death. Our screen led to the identification of several proteins that, when repressed, either sensitized or protected the cells from death resulting from unresolved ER stress. We confirmed 7 out of the 22 identified hits providing the strongest protection upon repression. We decided to further characterize the role of PRKAR1A, and found that its depletion specifically protected the cells from ER stressinduced apoptosis, and not from apoptosis induced by other means. PRKAR1A is a negative regulatory subunit of PKA, and we demonstrated that the protection obtained in cells depleted in PRKAR1A was the result of PKA activation. Accordingly, PKA activation by the adenylyl cyclase agonist Frsk similarly protected the cells from ER stress-induced apoptosis without affecting PRKAR1A levels. These results are in contrast with the reported proapoptotic role of PKA in various settings. Indeed, increased PKA activity has been reported to promote apoptosis by causing transcriptional induction and post-translational stabilization of BIM. ${ }^{29,34}$ In line with this, genetic deletion of Prkar1a leads to early embryonic lethality in mice, ${ }^{35,36}$ and its inducible loss in diverse adult tissues is reported to trigger PKA activation and apoptosis because of the upregulation of proapoptotic BCL-2 family members. ${ }^{15}$ Paradoxically, PKA is also reported to promote cell survival and its hyperactivation causes hereditary endocrine neoplasias. Indeed, heterogenic mutations in the human PRKAR1A gene, which leads to overactivation of the PKA signaling pathway, are strongly associated with a disorder called Carney complex, a rare autosomal dominant disorder characterized by hyperpigmentation of the skin, cardiac and other myxomas, endocrine tumors and schwannomas. ${ }^{12,17}$ The cellular conditions that regulate the pro- versus antiapoptotic role of PKA are not entirely understood. Here, we show that PKA has a prosurvival role in mammalian cells undergoing ER stress. It is therefore tempting to speculate that the prosurvival, prooncogenic, role of PKA in endocrine cells (highly secretory cells) may result from their higher basal levels of stress in the ER, owing to the higher demand for protein synthesis. Interestingly, the UPR is emerging as an important network contributing to the remodeling of cancer gene expression, thereby either preventing cell transformation or providing an advantage to transformed cells. ${ }^{37,38}$ Our results indicate that PKA had no effect on the activation of the UPR, and that its prosurvival role was, at least in part, independent of gene activation. Indeed, although protein translation inhibition by $\mathrm{CHX}$ partially protected the cells from ER stress-induced death, the protection was further enhanced when PKA was activated. These results therefore also exclude a role for the CREB transcription factor, a well-known substrate of PKA. Autophagy, a consequence of UPR activation known to have a protective role during ER stress, was not increased following PKA activation. Instead, we observed reduced autophagy induction in cells transiently activating PKA (siRNA against Prkar1a), which correlates with the reported role of PKA in the negative regulation of autophagy. ${ }^{39-41}$ This effect was, however, not observed in cells with a constitutively active PKA (genetic deletion), probably due to compensatory mechanisms.

MOMP is commonly considered as a point of no return during apoptosis. Mitochondrial fission, a process regulated by Drp1, has been reported to occur concomitantly with MOMP and cytochrome $c$ release. Although controversial, some reports indicate that fission is required for $B A X$ translocation into the mitochondrial membrane, cytochrome $c$ release and apoptosome assembly. ${ }^{9,42}$ Other studies have instead reported that Drp1 regulates MOMP independently of mitochondrial division, suggesting that Drp1 may regulate apoptosis by different means. ${ }^{32}$ PKA was shown to regulate mitochondrial fission and cell death by modulating Drp1 activity through phosphorylation. PKA inactivates human Drp1 through phosphorylation on Ser637, which abrogates its recruitment to the mitochondria. ${ }^{31} \mathrm{~A}$ recent study reported that MAPL-dependent SUMOylation of Drp1, which occurs downstream of PKA regulation, regulates the stability of the $\mathrm{ER} /$ mitochondrial contact sites, which are important for $\mathrm{Ca}^{2+}$ flux, cytochrome $c$ release and apoptosis induction. ${ }^{43}$ Interestingly, ER stress-induced BAX/BAK-dependent apoptosis has been associated with the release of ER calcium and concomitant increase of mitochondrial calcium. ${ }^{44}$ The mitochondrial $\mathrm{Ca}^{2+}$ overload generated leads to the depolarization 
of the inner membrane, the release of cytochrome $c$ release and the activation of caspases. ${ }^{45}$ In line with the reported occurrence of mitochondrial fission during ER stress ${ }^{46-48}$ and the role of Drp1 in ER stress-induced apoptosis of pancreatic $\beta$-cells, ${ }^{49}$ we found that PKA activation led to Drp1 inhibition, as monitored by Drp1 phosphorylation. Moreover, we demonstrated that PKA activation and Drp1 inhibition protected cells from apoptotosis induced by Tu but not by TNF or Eto, thereby identifying a direct mechanistic link for the protective effect of PKA activation in cells undergoing ER stress. It is important to note that the protection obtained by Drp1 repression/inhibition was not as potent as following PKA activation, suggesting that PKA additionally promotes cell survival by phosphorylating other target proteins. It was recently reported that AMPK also protects pancreatic $\beta$-cells and endothelial cells from ER stress-induced apoptosis through Drp1 phosphorylation, ${ }^{47,50}$ suggesting a common converging mechanism to protect cells from death under ER stress conditions. Another recent study performed in yeast indicates that PKA deactivation contributes to the prosurvival response to ER stress by inducing transcriptional upregulation of stress-related genes and repression of ribosome biogenesis genes. ${ }^{2}$ Our results therefore highlight clear differences between yeast and mammalian systems; nevertheless, it unmasks a central role of PKA during the UPR in eukaryotic cells.

Taken together, our results reveal the protective role of PKA activation in mammalian cells undergoing unresolved ER stress, thereby suggesting a potential therapeutic benefit of PKA-activating compounds for the treatment of pathologies resulting from excessive apoptosis caused by ER stress. Our results also bring potential new interpretations on the role of ER stress to the oncogenic role of PKA in the Carney complex disorder.

\section{Materials and Methods}

Antibodies and reagents. Antibodies were purchased from the following companies: anticleaved caspase-8 (Cell Signaling, Danvers, MA, USA; no. 9429), anti-caspase-3 (Cell Signaling; no. 9662), anti-caspase-9 (Cell Signaling; no. 9508), anticleaved PARP (poly-(ADP-ribose) polymerase) (Asp214) (Cell Signaling; no. 9544S), anti-Prkar1a (BD Transduction Laboratories, San Jose, CA, USA; no. 610166), anti-PKA (Cell Signaling; no. 4782), anti-IRE1 (Cell Signaling; no. 3294), anti-XBP1s (BioLegend, San Diego, CA, USA; no. 619501), anti-PERK (Cell Signaling; no. 3192), anti-phospho-elf2 $\alpha$ (Cell Signaling; no. 9721), anti-Bip (BD Transduction Laboratories; no. 610978), anti-CHOP (Cell Signaling; no. 2895), anti-LC3 (MBL, Woburn, MA, USA; no. PM036), anti-Drp1 (BD Transduction Laboratories; no. 611113), anti-phospho-Drp1 (Cell Signaling; no. 4867), anti- $\beta$ tubulin (Abcam, Cambridge, UK; no. ab6046-200). Tu was used at $1 \mu \mathrm{g} / \mathrm{ml}$ (SigmaAldrich, St Louis, MO, USA; no. T7765) and Th was used at $1 \mu \mathrm{M}$ (Sigma-Aldrich; no. T-9033). Recombinant human TNF- $\alpha$, produced and purified to at least $99 \%$ homogeneity in our laboratory, has a specific biological activity of $3 \times 10^{7} \mathrm{IU} / \mathrm{mg}$ and was used at $600 \mathrm{IU} / \mathrm{ml}(20 \mathrm{ng} / \mathrm{ml})$ to stimulate MEFs. The general protein translation inhibitor CHX (Sigma-Aldrich; no. C-7698) was used as $0.25 \mu \mathrm{g} / \mathrm{ml}$ (Figures 3a, b and $6 \mathrm{~g}$ ) and $1 \mu \mathrm{g} / \mathrm{ml}$ (Figure 6a). Eto (Enzo Life Sciences, Farmingdale, NY, USA; no. BML-GR307-0100) was used at a final concentration of $2 \mu \mathrm{M}$, H89 dihydrochloride (Calbiochem, Merck KGaA, Darmstadt, Germany; no. 371963) was used at $10 \mu \mathrm{M}$. Frsk (Tocris Bioscience, Bristol, UK; no. 1099) was used at $12 \mu \mathrm{M}$ and bafilomycin A1 (Sigma-Aldrich; no. B-1793) was used at $10 \mathrm{nM}$ and added $2 \mathrm{~h}$ before treatment with Tu. Mdivi-1 (Merck Chemicals NV, Overijse, Belgium; no. 475856) was used at $125 \mu \mathrm{M}$

Cell lines. SV40 large T-immortalized Ripk $1^{+/+}$MEFs (WT MEFs) have been described previously. ${ }^{19}$ Prkart ${ }^{\text {fl/fll }}$ MEFs were immortalized following the same protocol. HeLa and HEK293T cells were purchased from ATCC. The MEFs, HeLa and HEK293T cells were cultured in Dulbecco's modified Eagle's medium supplemented with $10 \%$ fetal calf serum and L-glutamine $(2 \mathrm{mM})$. Prkar1a ${ }^{\text {fl/t }}$ MEFs were transduced with adenovirus expressing EGFP or Cre-recombinase EGFP (Vector Biolabs, Malven, PA, USA; no. 1710) following the manufacturer's instruction. The cells were FACS sorted according to their extent of EGFP expression $48 \mathrm{~h}$ post-transduction. Prkar1a expression in the two cell populations was then analyzed by western blot analysis following standard protocol.

siRNA screen. MEFs were seeded at $2.5 \times 10^{3}$ cells per well in BD-imaging 96-well plates. The cells were transfected $8 \mathrm{~h}$ later with SMARTpool siRNAs (Dharmafect) (Supplementary Tables 1 and 2) using Dharmafect $1(0.2 \mu \mathrm{l}$ of Dharmafect and siRNA to a final concentration of $28.5 \mathrm{nM}$ per well). Each siRNAs was transfected in six different plates ( 1 well per plate). The media were changed $24 \mathrm{~h}$ post-transfection. The plates were stimulated with Tu $(1 \mu \mathrm{g} / \mathrm{ml})$ or DMSO, in triplicates, $48 \mathrm{~h}$ post-transfection. Cell death was measured $18 \mathrm{~h}$ later using a BDPathway 855 instrument (BD Biosciences, San Jose, CA, USA) equipped with an environmental control unit to ensure a constant temperature of $37^{\circ} \mathrm{C}$ and $5 \% \mathrm{CO}_{2}$ during image acquisition. Hoechst $33342(2 \mu \mathrm{g} / \mathrm{ml})$ (Invitrogen) and PI $(3 \mu \mathrm{M})$ (Sigma-Aldrich) were added to the cells $1 \mathrm{~h}$ before the analysis. Images were taken using a $\times 10$ objective (Olympus, NJ, USA) in a montage of $4 \times 4$, including at least 2000 cells per image and treatment condition (siRNA). Hoechst 33342 labeling was used to segment the nuclei and to extract Hoechst and PI intensity values of each nucleus, with the BD Attovision analysis software (BD Biosciences). The percentage of cell death was determined by substracting total PI-positive area out of total Hoescht area for every plate. The three plates treated with DMSO were analyzed similarly to evaluate the impact of the KD on cell viability. The percentage of cell death was normalized to the percentage of cell death per plate, using the NStreated cells as a reference.

Kinetic analysis of cell death. Cell death determined in a kinetic manner was carried out using a Fluostar Omega fluorescence plate reader (BMG Labtech, Ortenberg, Germany) with temperature- and atmosphere-controlled settings. MEFs were seeded in triplicate at 10000 cells per well in a 96-well adherent plate. The next day, cells were treated with the indicated compounds in the presence of $5 \mu \mathrm{M}$ SytoxGreen (Invitrogen; no. S-7020) and $10 \mu \mathrm{M}$ Ac-DEVD-AMC. SytoxGreen intensity was measured in function of the time at intervals of $1 \mathrm{~h}$ by using a Fluostar Omega fluorescence plate reader (BMG Labtech) with an excitation filter of $485 \mathrm{~nm}$, emission filter of $520 \mathrm{~nm}$, gains set at 1100, 20 flashes per well and orbital averaging with a diameter of $3 \mathrm{~mm}$. Cell death was calculated by subtracting the induced SytoxGreen fluorescence from the background fluorescence and by dividing the obtained result by the maximal fluorescence (minus the background fluorescence) obtained by permeabilization of the cells by using Triton X-100 at a final concentration of $0.1 \%$. Caspase-3 activity (cleaved DEVD-AMC) was obtained using an excitation filter of $355 \mathrm{~nm}$ and an emission filter of $460 \mathrm{~nm}$ with the same time intervals and gains of 1000 with 20 flashes per well and $3 \mathrm{~mm}$ orbital averaging. In case of siRNA-mediated repression, the smart pools siRNAs (Dharmafect) were transfected using Dharmafect 1 reagent following the manufacturer's instructions and the cell death trigger was added $48 \mathrm{~h}$ posttransfection.

Statistical analysis. Statistical analysis was performed with GraphPad Prism V6 software (GraphPad, La Jolla, CA, USA). Statistical significance between the indicated conditions was analyzed by multiple comparisons using two-way ANOVA (NS $=P>0.05 ;{ }^{*} P<0.05 ;{ }^{*} P<0.01$ ).

\section{Conflict of Interest}

The authors declare no conflict of interest.

Acknowledgements. We thank Inge Brugman for technical assistance and Lawrence S Kirschner (The Ohio State University, Columbus, OH, USA) for the kind gift of the Prkar1a ${ }^{f / f l}$ MEFs. MJMB has a tenure track position within the Multidisciplinary Research Program of Ghent University (GROUP-ID). Research in the Vandenabeele's unit is supported by Belgian Grants (IAP 7/32), Flemish Grants (FWO G087511, G097311, G0A4512N, G078713N, G017212N, and G013715N; Methusalem BOF09/01M00709), Ghent University Grants (MRP, GROUP-ID consortium), Grants from the Foundation Against Cancer (F94 and 2010-162) and Grants from the VIB. 
1. Hetz $C$. The unfolded protein response: controlling cell fate decisions under ER stress and beyond. Nat Rev Mol Cell Biol 2012; 13: 89-102.

2. Pincus D, Aranda-Diaz A, Zuleta IA, Walter P, El-Samad H. Delayed Ras/PKA signaling augments the unfolded protein response. Proc Natl Acad Sci USA 2014; 111: 14800-14805.

3. Jager R, Bertrand MJ, Gorman AM, Vandenabeele P, Samali A. The unfolded protein response at the crossroads of cellular life and death during endoplasmic reticulum stress. Biol Cell 2012; 104: 259-270.

4. Walter $P$, Ron $D$. The unfolded protein response: from stress pathway to homeostatic regulation. Science $2011 ; 334:$ 1081-1086.

5. Lu M, Lawrence DA, Marsters S, Acosta-Alvear D, Kimmig P, Mendez AS et al. Opposing unfolded-protein-response signals converge on death receptor 5 to control apoptosis. Science 2014; 345: 98-101.

6. Sano R, Reed JC. ER stress-induced cell death mechanisms. Biochim Biophys Acta 2013; 1833: 3460-3470.

7. Saveljeva S, Mc Laughlin SL, Vandenabeele P, Samali A, Bertrand MJ. Endoplasmic reticulum stress induces ligand-independent TNFR1-mediated necroptosis in L929 cells. Cell Death Dis 2015; 6: e1587.

8. Rodriguez D, Rojas-Rivera D, Hetz C. Integrating stress signals at the endoplasmic reticulum: the BCL-2 protein family rheostat. Biochim Biophys Acta 2011; 1813: 564-574.

9. Landes T, Martinou JC. Mitochondrial outer membrane permeabilization during apoptosis: the role of mitochondrial fission. Biochim Biophys Acta 2011; 1813: 540-545.

10. Verfaillie T, Garg AD, Agostinis P. Targeting ER stress induced apoptosis and inflammation in cancer. Cancer Lett 2013; 332: 249-264.

11. Lindholm D, Wootz H, Korhonen L. ER stress and neurodegenerative diseases. Cell Death Differ 2006; 13: 385-392.

12. Kirschner LS, Carney JA, Pack SD, Taymans SE, Giatzakis C, Cho YS et al. Mutations of the gene encoding the protein kinase A type I-alpha regulatory subunit in patients with the Carney complex. Nat Genet 2000; 26: 89-92.

13. Kirschner LS, Kusewitt DF, Matyakhina L, Towns WH II, Carney JA, Westphal $\mathrm{H}$ et al. A mouse model for the Carney complex tumor syndrome develops neoplasia in cyclic AMP-responsive tissues. Cancer Res 2005; 65: 4506-4514.

14. Kirschner LS, Sandrini F, Monbo J, Lin JP, Carney JA, Stratakis CA. Genetic heterogeneity and spectrum of mutations of the PRKAR1A gene in patients with the carney complex. Hum Mol Genet 2000; 9: 3037-3046.

15. Gangoda L, Doerflinger M, Srivastava R, Narayan N, Edgington LE, Orian J et al. Loss of Prkar1a leads to Bcl-2 family protein induction and cachexia in mice. Cell Death Differ 2014 21: $1815-1824$.

16. Carney JA. Carney complex: the complex of myxomas, spotty pigmentation, endocrine overactivity, and schwannomas. Semin Dermatol 1995; 14: 90-98.

17. Carney JA. The Carney complex (myxomas, spotty pigmentation, endocrine overactivity, and schwannomas). Dermatol Clin 1995; 13: 19-26.

18. Estornes Y, Aguileta MA, Dubuisson C, De Keyser J, Goossens V, Kersse K et al. RIPK1 promotes death receptor-independent caspase-8-mediated apoptosis under unresolved ER stress conditions. Cell Death Dis 2014; 5: e1555.

19. Dondelinger Y, Aguileta MA, Goossens V, Dubuisson C, Grootjans S, Dejardin E et al. RIPK3 contributes to TNFR1-mediated RIPK1 kinase-dependent apoptosis in conditions of clAP1/2 depletion or TAK1 kinase inhibition. Cell Death Differ 2013; 20: 1381-1392.

20. Elyaman W, Terro F, Suen KC, Yardin C, Chang RC, Hugon J. BAD and Bcl-2 regulation are early events linking neuronal endoplasmic reticulum stress to mitochondria-mediated apoptosis. Brain Res Mol Brain Res 2002; 109: 233-238.

21. Li J, Lee B, Lee AS. Endoplasmic reticulum stress-induced apoptosis: multiple pathways and activation of p53-up-regulated modulator of apoptosis (PUMA) and NOXA by p53. J Biol Chem 2006; 281: 7260-7270.

22. Nadella KS, Jones GN, Trimboli A, Stratakis CA, Leone G, Kirschner LS. Targeted deletion of Prkar1a reveals a role for protein kinase $A$ in mesenchymal-to-epithelial transition. Cancer Res 2008; 68: 2671-2677.

23. Hu P, Han Z, Couvillon AD, Kaufman RJ, Exton JH. Autocrine tumor necrosis factor alpha links endoplasmic reticulum stress to the membrane death receptor pathway through IRE1alpha-mediated NF-kappaB activation and down-regulation of TRAF2 expression. Mol Cell Biol 2006; 26: 3071-3084.

24. Skalhegg BS, Tasken K. Specificity in the CAMP/PKA signaling pathway. Differential expression, regulation, and subcellular localization of subunits of PKA. Front Biosci 2000; 5 : D678-D693.

25. Pidoux G, Tasken K. Specificity and spatial dynamics of protein kinase A signaling organized by A-kinase-anchoring proteins. J Mol Endocrinol 2010; 44: 271-284.
26. Tasken K, Aandahl EM. Localized effects of cAMP mediated by distinct routes of protein kinase A. Physiol Rev 2004; 84: 137-167.

27. Coley AM, Moujalled D, Puthalakath H. The PKA paradox: is Bim the answer? Cell Cycle 2011; 10: 729-730

28. Chaturvedi D, Cohen MS, Taunton J, Patel TB. The PKARlalpha subunit of protein kinase A modulates the activation of p90RSK1 and its function. J Biol Chem 2009; 284: 23670-23681.

29. Moujalled D, Weston R, Anderton H, Ninnis R, Goel P, Coley A et al. Cyclic-AMP-dependent protein kinase $\mathrm{A}$ regulates apoptosis by stabilizing the $\mathrm{BH} 3$-only protein Bim. EMBO Rep 2011; 12: 77-83.

30. Ishihara N, Nomura M, Jofuku A, Kato H, Suzuki SO, Masuda K et al. Mitochondrial fission factor Drp1 is essential for embryonic development and synapse formation in mice. Nat Cell Biol 2009; 11: 958-966.

31. Cribbs JT, Strack S. Reversible phosphorylation of Drp1 by cyclic AMP-dependent protein kinase and calcineurin regulates mitochondrial fission and cell death. EMBO Rep 2007; 8: 939-944.

32. Cassidy-Stone A, Chipuk JE, Ingerman E, Song C, Yoo C, Kuwana T et al. Chemical inhibition of the mitochondrial division dynamin reveals its role in Bax/Bak-dependent mitochondrial outer membrane permeabilization. Dev Cell 2008; 14: 193-204.

33. Kim I, Xu W, Reed JC. Cell death and endoplasmic reticulum stress: disease relevance and therapeutic opportunities. Nat Rev Drug Discov 2008; 7: 1013-1030.

34. Lee YY, Moujalled D, Doerflinger M, Gangoda L, Weston R, Rahimi A et al. CREB-binding protein $(\mathrm{CBP})$ regulates beta-adrenoceptor (beta-AR)-mediated apoptosis. Cell Death Differ 2013; 20: 941-952.

35. Amieux PS, Howe DG, Knickerbocker H, Lee DC, Su T, Laszlo GS et al. Increased basal cAMP-dependent protein kinase activity inhibits the formation of mesoderm-derived structures in the developing mouse embryo. J Biol Chem 2002; 277: 27294-27304.

36. Kirschner LS, Yin Z, Jones GN, Mahoney E. Mouse models of altered protein kinase A signaling. Endocr Relat Cancer 2009; 16: 773-793.

37. Chevet E, Hetz C, Samali A. Endoplasmic reticulum stress-activated cell reprogramming in oncogenesis. Cancer Discov 2015; 5: 586-597.

38. Kato $\mathrm{H}$, Nishitoh $\mathrm{H}$. Stress responses from the endoplasmic reticulum in cancer. Front Oncol 2015; 5 : 93.

39. Cherra SJ III, Kulich SM, Uechi G, Balasubramani M, Mountzouris J, Day BW et al. Regulation of the autophagy protein LC3 by phosphorylation. J Cell Biol 2010; 190: 533-539.

40. Mavrakis M, Lippincott-Schwartz J, Stratakis CA, Bossis I. mTOR kinase and the regulatory subunit of protein kinase A (PRKAR1A) spatially and functionally interact during autophagosome maturation. Autophagy 2007; 3: 151-153.

41. de Joussineau C, Sahut-Barnola I, Tissier F, Dumontet T, Drelon C, Batisse-Lignier M et al. mTOR pathway is activated by PKA in adrenocortical cells and participates in vivo to apoptosis resistance in primary pigmented nodular adrenocortical disease (PPNAD). Hum Mol Genet 2014; 23: 5418-5428.

42. Youle RJ, van der Bliek AM. Mitochondrial fission, fusion, and stress. Science 2012; 337: 1062-1065.

43. Prudent J, Zunino R, Sugiura A, Mattie S, Shore GC, McBride HM. MAPL SUMOylation of Drp1 stabilizes an ER/mitochondrial platform required for cell death. Mol Cell 2015; 59: 941-955.

44. Nutt LK, Pataer A, Pahler J, Fang B, Roth J, McConkey DJ et al. Bax and Bak promote apoptosis by modulating endoplasmic reticular and mitochondrial $\mathrm{Ca}^{2+}$ stores. J Biol Chem 2002; 277: 9219-9225.

45. Malhotra JD, Kaufman RJ. ER stress and its functional link to mitochondria: role in cell survival and death. Cold Spring Harbor Perspect Biol 2011; 3: a004424.

46. Bhavya BC, Indira D, Seervi M, Joseph J, Sobhan PK, Mathew KA et al. Endoplasmic reticulum-targeted $\mathrm{Bcl}-2$ inhibitable mitochondrial fragmentation initiates ER stress-induced cell death. Adv Exp Med Biol 2012; 749: 83-95.

47. Wikstrom JD, Israeli T, Bachar-Wikstrom E, Swisa A, Ariav Y, Waiss M et al. AMPK regulates ER morphology and function in stressed pancreatic beta-cells via phosphorylation of DRP1. Mol Endocrinol 2013; 27: 1706-1723.

48. Hom JR, Gewandter JS, Michael L, Sheu SS, Yoon Y. Thapsigargin induces biphasic fragmentation of mitochondria through calcium-mediated mitochondrial fission and apoptosis. J Cell Physiol 2007; 212: 498-508.

49. Peng L, Men X, Zhang W, Wang H, Xu S, Xu M et al. Dynamin-related protein 1 is implicated in endoplasmic reticulum stress-induced pancreatic beta-cell apoptosis. Int J Mol Med 2011; 28: 161-169.

50. Li J, Wang Y, Wang Y, Wen X, Ma XN, Chen W et al. Pharmacological activation of AMPK prevents Drp1-mediated mitochondrial fission and alleviates endoplasmic reticulum stressassociated endothelial dysfunction. J Mol Cell Cardiol 2015; 86: 62-74. 